\title{
La mesure de l'excrétion urinaire de l'uranium par spectrofluorimétrie laser à résolution temporelle
}

\author{
D. CAVADORE* , B. POIREY* , J.B. COMBA*, G. MINAUD*, D. BALLET **
}

(Manuscrit reçu le 30 septembre 1998, accepté le 10 décembre 1998)

RÉSUMÉ La quantification directe rapide de l'excrétion urinaire de l'uranium est souvent perturbée par des incertitudes métaboliques et par des interférences analytiques. Ces phénomènes conduisent à des limites de détection ou à des incertitudes trop élevées. La technique proposée associe à la fois un traitement rapide de l'échantillon et un système optimisé de mesure. L'association d'un laser solide de puissance utilisé comme source d'excitation et d'un fluorimètre modifíé du commerce permet d'atteindre les objectifs de l'étude : rapidité de réponse et facilité de mise en euvre, précision et exactitude inférieures à $10 \%$. Les stades analytiques selon deux modalités (mesure directe et après minéralisation de l'échantillon) sont décrits. Les résultats expérimentaux portant sur 120 mesures sont comparés aux résultats obtenus par chromatographie d'extraction. Les avantages et inconvénients de la technique sont commentés. Enfin les valeurs de l'excrétion urinaire naturelle de l'uranium chez 80 travailleurs non-exposés de la région de Marcoule sont présentées en fonction de la technique analytique choisie.

ABSTRACT The measurement of the urinary excretion of uranium by time-resolved laser-induced fluorescence (TRLIF).

Rapid direct measurements of the urinary excretion of uranium are often disturbed by metabolic uncertainties and analytical interferences. One consequence of these phenomena is detection limits or uncertainties that are too high. The technique proposed here associates rapid processing of the sample with an optimised measurement system. The objectives of the study - rapidity of response, accuracy and precision lower than $10 \%$ and ease of operation - are attained by using a solid power laser as excitation source in conjunction with a modified commercial fluorimeter. We describe the analytical stages for the two methods used (direct measurement and measurement after mineralisation of the sample). The experimental results achieved with 120 measurements are compared with the results obtained by extraction chromatography. The advantages and drawbacks of the TRLIF technique are discussed. Finally, the values of the natural urinary excretion of uranium among 80 non-exposed workers from the Marcoule region are presented as a function of the analytical technique selected.

\footnotetext{
COGEMA, Établissement de Marcoule, Laboratoire d'Analyses Médicales, 30206 Bagnols-sur-Cezze Cedex, France.

** Université de Rennes 1, Chimie du Solide et Inorganique Moléculaire, 35042 Rennes Cedex, France.
} 


\section{Introduction}

La surveillance de la contamination interne des travailleurs exposés à des composés de l'uranium s'effectue à la fois par la mesure des raies $\mathrm{X}$ et gamma des isotopes de l'uranium ou de ses descendants radioactifs, déposés au niveau de l'arbre trachéo-bronchique, et/ou par la mesure de l'excrétion urinaire et/ou fécale de ces mêmes isotopes.

La mesure directe de l'incorporation des divers isotopes de l'uranium par spectrométrie pulmonaire est une mesure certes indispensable, mais rendue difficile par le fait que la matière vivante absorbe partiellement certaines raies gamma et totalement certaines raies $\mathrm{X}$ conduisant à des limites de détection très variables selon la morphologie des sujets mesurés. De plus, cette mesure ne fournit que peu d'informations sur la composition isotopique du contaminant. C'est pourquoi elle doit être complétée par des mesures d'excrétion qui sont libérées de ce type de contraintes.

Parmi les méthodes utilisables tant en situation de routine qu'en situation d'incident, deux sont utilisées dans notre laboratoire : la mesure de l'activité alpha des isotopes de l'uranium après séparation radiochimique par chromatographie d'extraction et la mesure directe de la masse d'uranium par spectrofluorimétrie laser à résolution temporelle (SLRT). Ces deux méthodes se complètent et permettent d'apprécier à la fois le risque radiotoxique et le risque chimiotoxique de l'uranium éventuellement incorporé par le personnel exposé.

La mesure directe de l'uranium par fluorimétrie se heurte à un certain nombre de difficultés :

- l'uranium métabolisé est éliminé dans les urines sous diverses formes chimique : bicarbonate d'uranyle, uranium lié à des protéines ou uranium sous forme chimique inconnue;

- les mesures par fluorimétrie traditionnelle sont sensibles aux fluorescences organiques parasites et nécessitent un traitement chimique de l'échantillon;

- les mesures par SLRT sont sensibles à la présence d'ions inhibiteurs (nitrate, chlorure, ...).

L'analyse idéale doit donc rassembler les qualités suivantes :

- rapidité d'exécution pour appréhender au plus vite le risque chimiotoxique sur le rein;

- reproductibilité;

- facilité de mise en œuvre. 


\section{Matériel et méthodes}

\subsection{Dosage de l'uranium par Chromatographie d'extraction} (Harduin et al., 1994; Horwitz, 1990; Horwitz, 1992; Nelson, 1990)

Après ajout de ${ }^{232} \mathrm{U}$, traceur purifié (Still, 1974) pour éliminer son premier descendant ${ }^{228} \mathrm{Th}$, l'échantillon d'urine est évaporé en présence d'acide nitrique $14,4 \mathrm{M}$ (Merck) et de peroxyde d'hydrogène à $30 \%$ (Carlo Erba), puis minéralisé au four à moufles pendant 12 heures. Après mise en solution du résidu, les poly-phosphates sont hydrolysés en milieu acide nitrique 1,44 M. Le précipité de phosphates alcalino-terreux, obtenu en alcalinisant le milieu, est ensuite dissous en milieu acide nitrique $1 \mathrm{M}$, en présence de nitrate d'aluminium. La séparation chromatographique s'effectue par chromatographie d'extraction sur des colonnes TEVA ${ }^{(8)}$ et TRUSpec ${ }^{(B)}$ (Eichrom). Après élution, l'échantillon est co-précipité avec du fluorure de lanthane $(100 \mu \mathrm{g})$ et séparé sur membrane d'ester de cellulose. L'activité alpha de l'échantillon est mesurée sur des compteurs à scintillation solide au sulfure de zinc activé à l'argent ( $\mathrm{ZnS}(\mathrm{Ag}))$ (Novelec) pendant 15 heures, suivie d'une spectrométrie alpha sur barrières de surface à ultra-bas bruit de fond (EG\&G Ortec) pendant 72 heures.

\subsection{Mesure de la masse d'uranium PAR SLRT}

Deux types de mesure sont systématiquement pratiquées :

- la mesure rapide sur l'échantillon d'urine non minéralisée;

- la mesure différée sur l'échantillon d'urine après minéralisation.

Pour pallier les difficultés rencontrées dans la mesure directe de la masse d'uranium par SLRT, nous avons été amenés à développer une nouvelle technique originale permettant d'éliminer de l'échantillon d'urine la majorité des composés organiques fluorescents ainsi qu'un certain nombre d'inhibiteurs de la fluorescence de l'uranium présents en grande quantité dans les urines.

En jouant sur les formes chimiques des différents complexes de l'uranium (VI) en fonction du $\mathrm{pH}$ en milieu carbonate (Fuger, 1992), il est possible de séparer l'uranium des composés inhibiteurs de fluorescence présents en grande quantité dans l'urine.

En analysant la figure 1, on constate que l'uranium (VI) pour des valeurs de $\mathrm{pH}<3$ se présente à $100 \%$ sous forme d'ion $\mathrm{UO}_{2}{ }^{2+}$ et à $\mathrm{pH}>9$ il se présente à $100 \%$ sous forme d'un complexe carbonate $\left[\mathrm{UO}_{2}\left(\mathrm{CO}_{3}\right)_{3}\right]^{4}$. Ce complexe est un des complexes de l'uranium (VI) dont la constante apparente de complexation est une des plus fortes connues à ce jour. Pour les valeurs de $\mathrm{pH}$ intermédiaires, de très nombreux complexes différents coexistent. En particulier, dans la zone de $\mathrm{pH}$ 
comprise entre 5 et 7 ( $\mathrm{pH}$ habituels d'émission de l'urine physiologique), on note la présence de complexes hydroxo et carbonato de l'uranium (VI), expliquant la difficulté de la mesure directe de l'uranium (VI), ces complexes se prêtant mal à la formation d'un fluorophore mesurable par fluorimétrie.

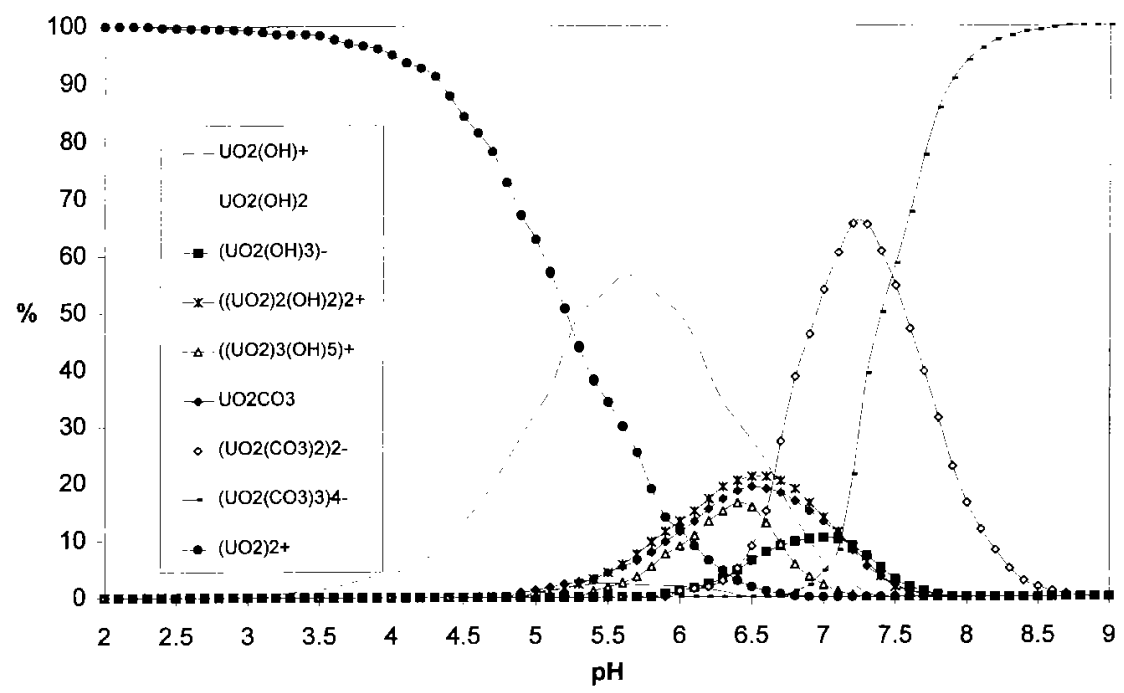

Fig. I-Diagramme de spéciation de l'uranium (VI). Conditions : [U] $10 \mu \mathrm{gl} \mathrm{l}^{-1}, \mathrm{NaClO}_{4} \mathrm{O}, \mathrm{I} \mathrm{M}, \mathrm{pCO}$, $10^{25} \mathrm{~atm}$.

Speciation diagram of uranium (VI). Conditions: [U] $10 \mu \mathrm{gL}^{-I}, \mathrm{NaClO}_{4} 0.1 \mathrm{M}, \mathrm{PCO}_{2}$ $10^{25}$ atm.

Le diagramme de spéciation présenté, fourni à titre indicatif, a servi de base de réflexion pour mener une stratégie de mise au point technique du dosage de l'uranium dans les urines par SLRT, en ne perdant pas de vue la présence possible dans la matrice urinaire de complexes sensiblement différents de ceux présents en milieu non-complexant. C'est pourquoi en posant l'hypothèse que l'uranium métabolisé et excrété dans l'urine - milieu très complexant - a un comportement analogue à l'uranium en milieu peu complexant, tel que celui présenté dans les diagrammes de spéciation, il s'est avéré possible de mesurer quantitativement l'uranium présent dans ce milieu moyennant quelques étapes simples de préparation chimique de l'échantillon.

\subsection{Chimie préparative}

L'échantillon d'urine centrifugé est préalablement filtré sur membrane d'ester de cellulose pour éliminer les débris cellulaires. La matière organique soluble va ensuite être éliminée par adsorption sur du charbon actif. Le charbon actif utilisé pour éliminer la matière organique présente l'inconvénient de retenir la majeure partie de l'ion $\mathrm{UO}_{2}^{2+}$ forme libre. C'est pourquoi l'uranium sera complexé préalablement par ajout d'un 
large excès de carbonate de sodium (Prolabo) sous forme d'un complexe $\left[\mathrm{UO}_{2}\left(\mathrm{CO}_{3}\right)_{3}\right]^{4-}$, forme sous laquelle il n'est pas retenu par le charbon actif.

À l'échantillon est ajoutée de la poudre de charbon actif, qui est ensuite agité magnétiquement. Le charbon est ensuite séparé par filtration, le filtrat contenant l'uranium complexé.

La très grande stabilité du complexe carbonate de l'uranium (VI) rend celui-ci non-mesurable directement par fluorimétrie. Il convient au préalable de dissocier le complexe, en acidifiant le milieu à $\mathrm{pH}<2$, ce qui permet de libérer l'ion UO22+. La mesure par SLRT est alors possible après ajout de Fluran ${ }^{\circledR}$, agent complexant spécifique formant un fluorophore, exacerbant la fluorescence de l'uranium, dans les conditions opératoires définies (Fluran ${ }^{\circledR} 10 \%$, milieu tamponné pH 5).

\subsection{Mesure par SLRT}

\subsubsection{Appareillage}

La SLRT a été utilisée depuis quelques années dans de nombreuses applications de dosages d'actinides ou de terres rares par mesures de fluorescence (Berthoud, 1988; Deniau, 1993; Moulin, 1990; Moulin, 1995). Son application au dosage de l'uranium dans les urines est relativement récente (Decambox, 1991).

Nous avons sélectionné comme source d'excitation un laser de puissance de type Nd-YAG dont la raie initiale dans l'infrarouge (1 $052 \mathrm{~nm})$ est transformée en une raie à $355 \mathrm{~nm}$ après ajout de cristaux doubleur et tripleur (Laser Brilliant ${ }^{\circledR}$, Quantel). Ce type de laser standard fournit une impulsion de 5,7 ns à une fréquence de $50 \mathrm{~Hz}$ et délivre par impulsion une énergie de $2 \mathrm{~J}$ (Moulin, 1994). Cette énergie considérable a dû être bridée à $28 \mathrm{~mJ}$ par pulse de façon à éviter la vaporisation de la solution, la formation de plasma dans la solution et la destruction des structures du fluorimètre.

Ce laser est couplé à un spectrofluorimètre (Fluo 2001 ${ }^{\circledR}$, Dilor) qui recueille par un polychromateur à $90^{\circ}$ par rapport au faisceau excitateur, le spectre de fluorescence émis. L'analyse du spectre s'effectue à travers un réseau holographique sur une barrette de photodiodes intensifiées et refroidies par effet Peltier $\left(-30{ }^{\circ} \mathrm{C}\right)$. L'enregistrement du spectre est mené en intégrant le signal pulsé obtenu en sortie de l'intensificateur pendant des temps variables selon la limite de détection désirée.

Des modifications de l'appareillage standard ont été effectuées dans notre laboratoire pour abaisser le bruit de fond :

- ajout d'un piège à lumière sous forme d'un tube dans le prolongement du faisceau laser, qui capte tous les rayons réfléchis par la cuve de mesure et les structures en aluminium du porte-cuve;

- apport d'eau à $5^{\circ} \mathrm{C}$ grâce à un cryostat à circulation ; 
- monture du système laser sur une table optique équipée d'un train de prismes améliorant la pureté spectrale du pulse d'excitation à $355 \mathrm{~nm}$, en élimipant la raie à $532 \mathrm{~nm}$ provenant du cristal doubleur (Fig. 2 et 3 ).

\subsubsection{Méthode}

Le but de notre étude était de comparer les résultats du dosage radiochimique de l'uranium par chromatographie d'extraction et ceux obtenus par SLRT selon deux modalités : mesure rapide directe et mesure sur minéralisats d'échantillons d'urine.

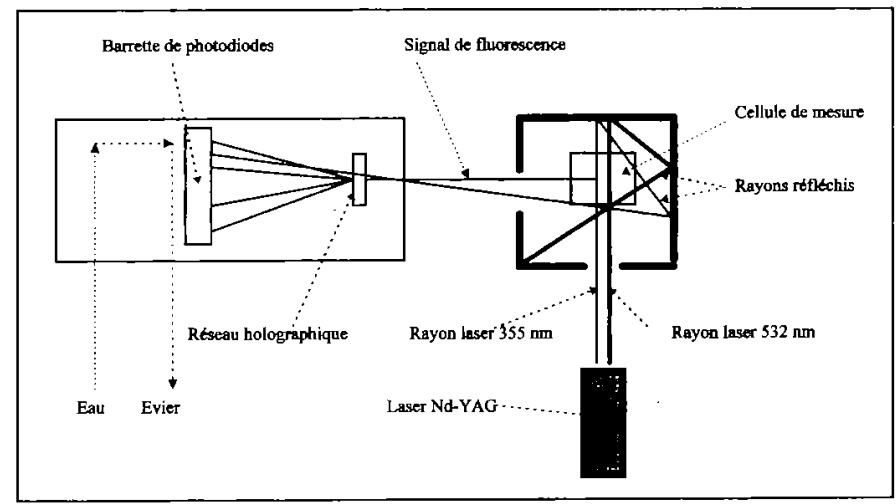

Fig. 2 - Représentation schématique de l'appareillage commercial de SLRT.

Schematic representation of TRLIF commercial apparatus.

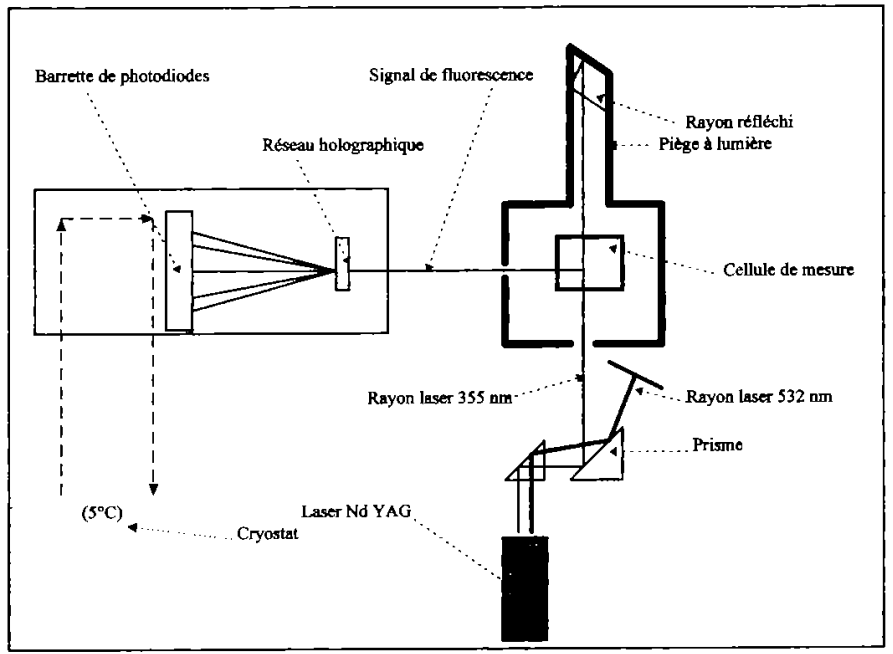

Fig. 3 - Représentation schématique de l'appareillage commercial de SLRT modifié. Schematic representation of TRLIF modified commercial apparatus. 
Les deux méthodes décrites ci-dessus ont été pratiquées sur 120 échantillons urinaires provenant soit d'incidents de contaminations, soit de prélèvements effectués au cours de la surveillance systématique de travailleurs professionnellement exposés, soit de prélèvements de personnels non-exposés.

Toutes les mesures effectuées en SLRT ont été menées par la méthode des ajouts dosés, ce qui permet de s'affranchir de l'influence des agents inhibiteurs de fluorescence, organiques ou minéraux, pouvant être présents dans les échantillons d'urine.

Le calcul de la concentration urinaire s'effectue par interpolation de la droite obtenue par régression linéaire par la méthode des moindres carrés, à partir des valeurs moyennées des signaux obtenus avant et après au moins trois ajouts dosés.

Le schéma de synthèse des deux méthodes utilisées dans cette étude est présenté figure 4 .

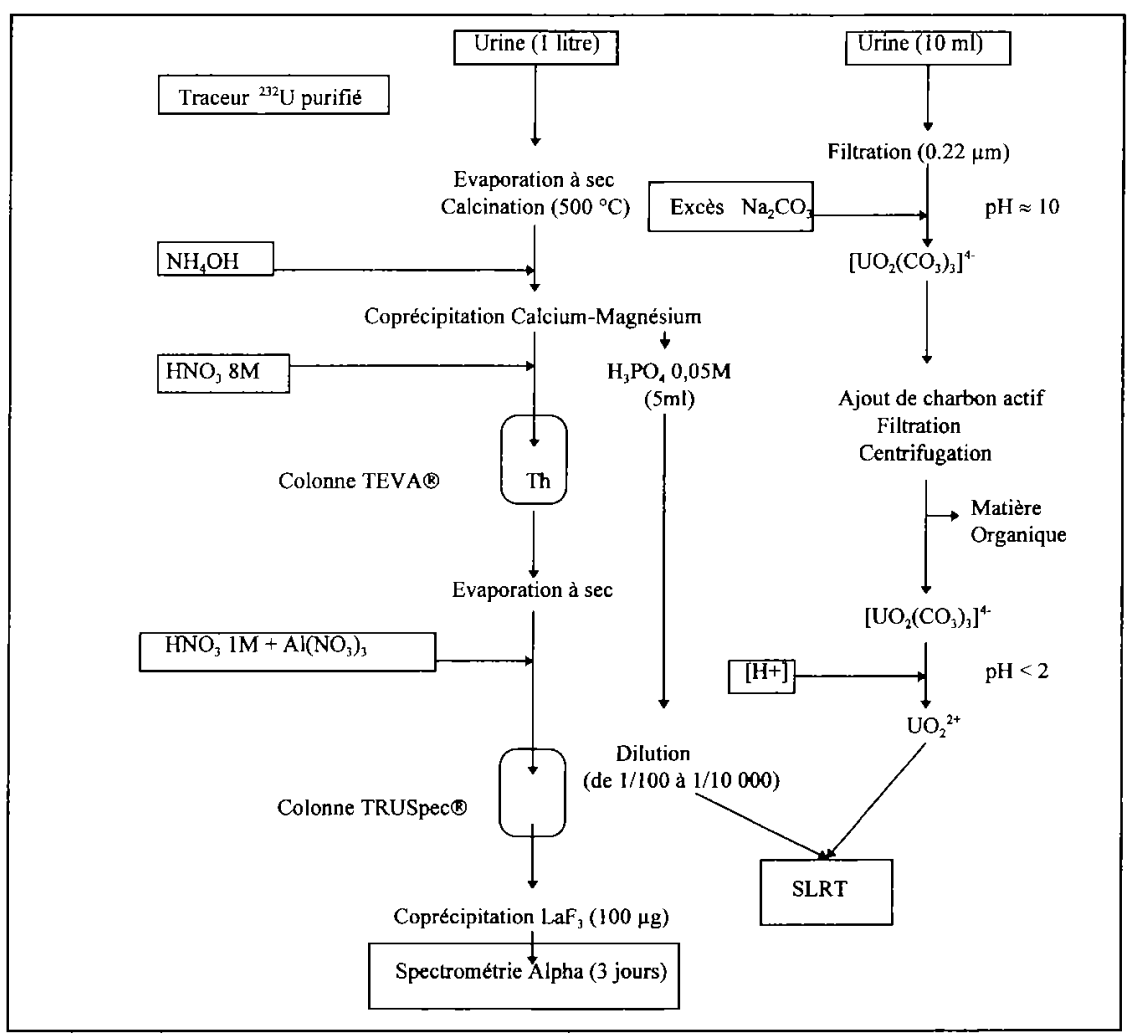

Fig. 4 - Étapes schématiques du dosage de l'uranium dans les urines par chromatographie d'extraction et par SLRT.

Schematic steps for urinary uranium measurements by extraction chromatography and TRLIF. 


\section{Résultats}

\subsection{Spectre de fluorescence de l'uranium}

La figure 5 représentant le spectre de fluorescence obtenu pour la même urine surchargée à 2 niveaux $\left(0,2 \mu \mathrm{g} \mathrm{l}^{-1}\right.$ et $\left.2,0 \mu \mathrm{g} \mathrm{l}^{-1}\right)$, montre les 4 pics caractéristiques de l'ion uranyle $(499,520,545$ et $570 \mathrm{~nm})$ pour des temps d'intégration de la mesure, respectivement de 3 fois 20 secondes et de 3 fois 10 secondes.

Le réglage du fluorimètre doit être effectué en ouvrant la porte de mesure quelques microsecondes après l'émission de l'impulsion laser, de façon à ne mesurer que la fluorescence spécifique de l'ion uranyle à longue durée de vie (>50 $\mu$ s) en éliminant les fluorescences organiques parasites (de l'ordre de la microseconde).

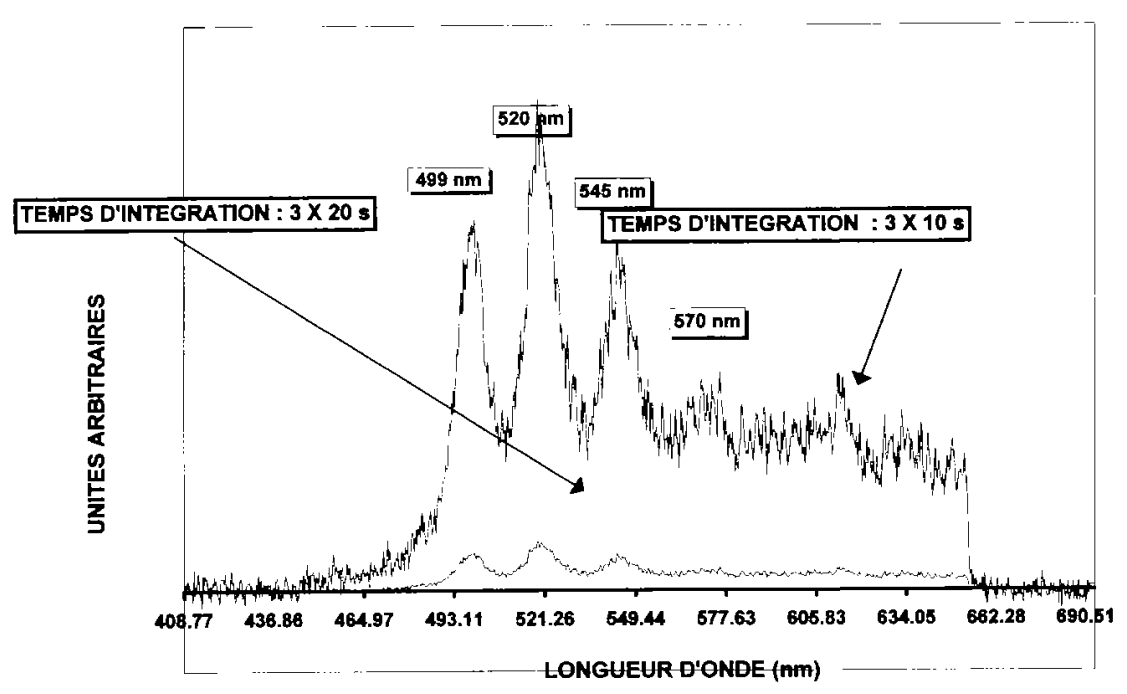

Fig. 5 - Spectre de fluorescence de l'uranium - Concentrations urinaires : $2,0 \mu \mathrm{g} t^{-1}$ et $0,2 \mu g t^{1}$ Conditions : milieu FLURAN ${ }^{\otimes} 10 \%$.

Uranyl fluorescence spectrum - Urinary concentrations : $2.0 \mu \mathrm{g} \mathrm{L} L^{-1}$ and $0.2 \mu \mathrm{g} L^{-1}$ Conditions: medium FLURAN ${ }^{\otimes} 10 \%$.

\subsection{Courbes}

La figure 6 présente les masses d'uranium obtenues pour chaque échantillon à la fois par méthode radio-chimique et par SLRT sur les échantillons d'urine minéralisés.

La figure 7 présente les masses d'uranium obtenues pour chaque échantillon à la fois par méthode radio-chimique et par SLRT sur les échantillons d'urine non minéralisées traités selon notre mode opératoire rapide. 
LA MESURE DE L'EXCRÉTION URINAIRE DE L'URANIUM

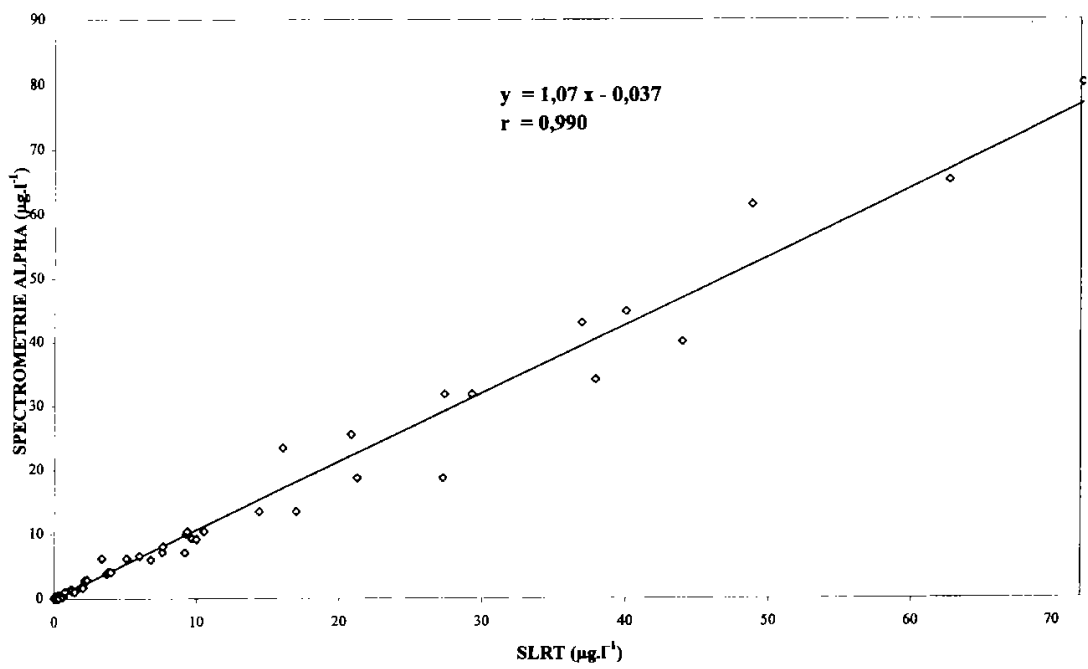

Fig. 6 - Comparaison des mesures par spectrométrie alpha et par SLRT (urines minéralisées).

Comparison of measurements performed by alpha spectrometry and by TRLIF (Mineralized urines).

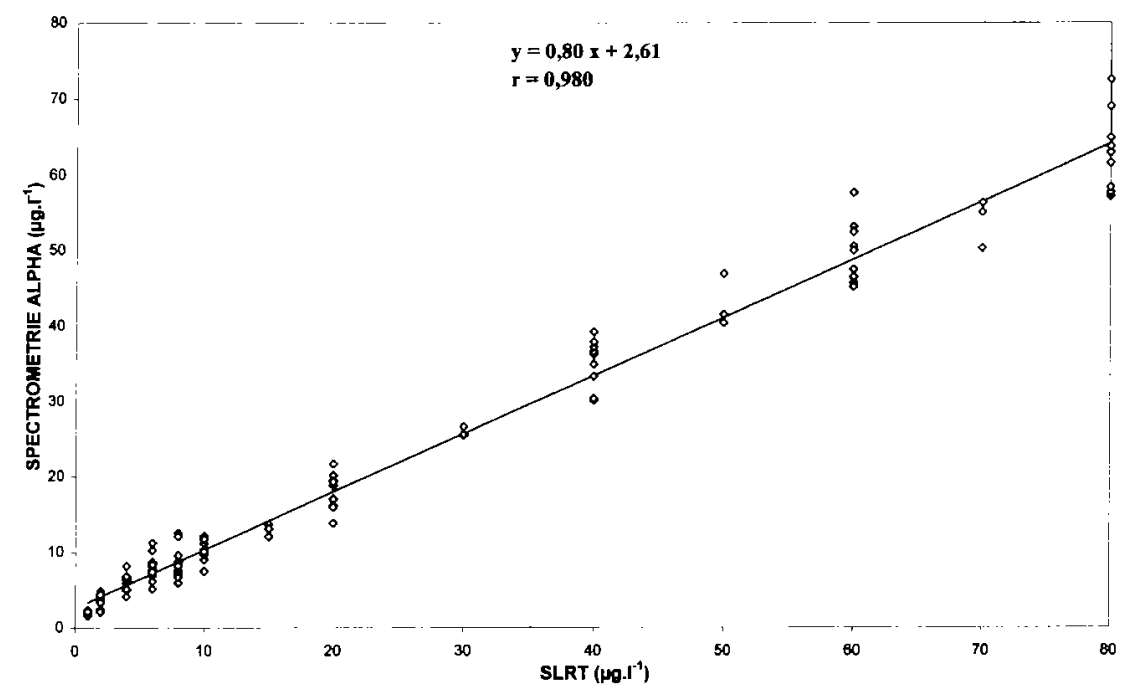

Fig. 7 - Comparaison des mesures par spectrométrie alpha et par SLRT (urines non minéralisées traitées au charbon actif).

Comparison of measurement performed by alpha spectrometry and by TRLIF (non-mineralized urines treated with activated carbon). 


\section{Discussion}

\section{1. Étude de la correlation des deux méthodes}

Les équations des droites d'ajustements obtenues par régression linéaire selon la méthode des moindres carrés sont illustrées dans le tableau I.

TABLEAU I

Équations des droites de régréssion et coefficients de corrélation. Straight line and correlation coefficient equations.

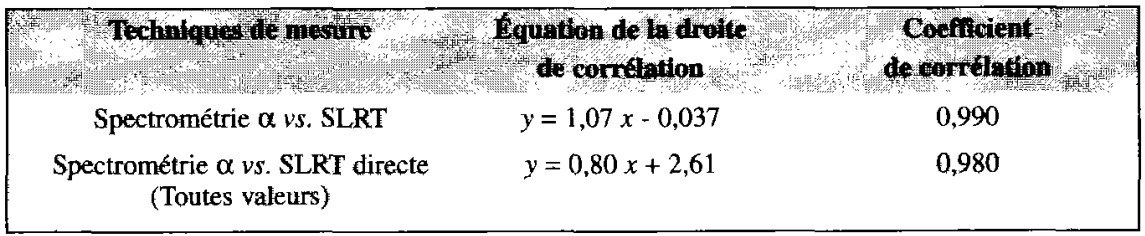

Les coefficients de corrélation, obtenus en comparant la SLRT à la technique de référence par spectrométrie alpha après séparation radiochimique, confirment la validité de la méthode fluorimétrique proposée pour le dosage de l'uranium, sur les échantillons minéralisés ou non.

\subsection{Exactitude - précision}

Le fluorimètre utilisé permet d'enregistrer individuellement les différentes intégrations du signal sur des durées illimitées. En utilisant cette possibilité nous avons procédé à des mesures d'exactitude en dosant 50 fois le même échantillon surchargé avec de l'uranium naturel à raison de $1 \mu \mathrm{g} \mathrm{l}^{-1} \mathrm{~d}^{\prime}$ urine, en effectuant de 1 à 5 intégrations pour chaque mesure selon les deux protocoles testés : dosage sur urines minéralisées et dosage sur urines traitées au charbon.

Les résultats sont présentés dans les tableaux II et III.

\section{TABLEAU II}

Exactitude et précision en fonction du nombre de mesures (Urines minéralisées).

Precision and accuracy as a function of the number of measurements (Mineralized urines).

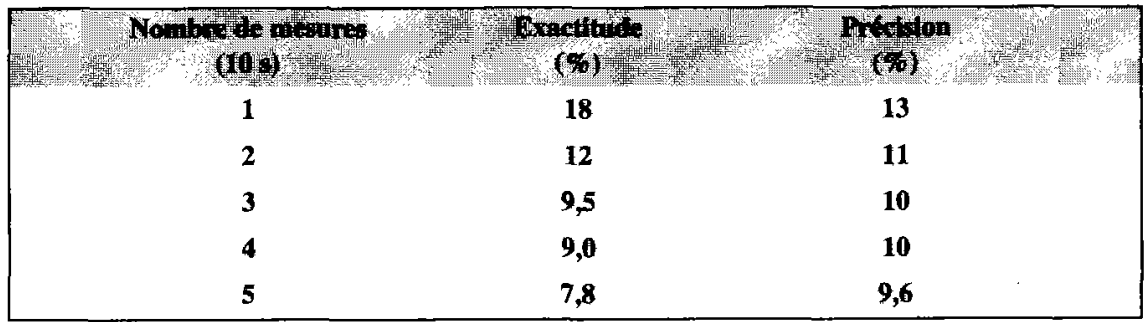


TABLEAU III

Exactitude et précision en fonction du nombre de mesures (urines non-minéralisées traitées sur charbon actif).

Precision and accuracy as a function of the number of measurements (non-mineralized urines).

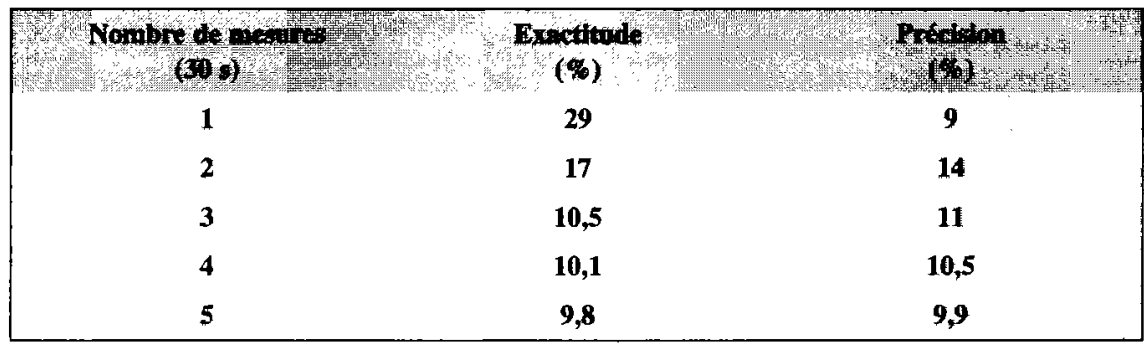

Précision et exactitude sont très proches de $10 \%$ dans les deux protocoles de mesures étudiés et sont conformes aux objectifs fixés pour ces dosages $(<10 \%$ pour des temps de mesures respectifs de 30 secondes et de 90 secondes).

\subsection{Valeurs normales de l'excrétion de l'uranium}

En parallèle, nous avons exploité les résultats de 80 dosages d'uranium chez une population de travailleurs non exposés à des risques de contamination interne, de façon à déterminer quelle était l'excrétion moyenne naturelle de l'uranium apporté par l'alimentation, dans la partie Est du département du Gard et la partie Ouest du département du Vaucluse (France).

Toutes les valeurs inférieures à $1 \mu \mathrm{g} 1^{-1}$, obtenues à la fois par séparation par chromatographie d'extraction associée à une spectrométrie alpha et par SLRT sur minéralisats d'urines ont été collectées et sont représentées sur la figure 8.

Les résultats obtenus sont en majeure partie inférieurs à $0,4 \mu \mathrm{g}^{-1}$. Les quelques valeurs au-dessus de cette limite sont dues à des excrétions d'uranium consécutives à la consommation d'eau minérale uranifère. Cela a été confirmé par le déséquilibre isotopique retrouvé, parfaitement identifié dans certaines eaux minérales (Remy, 1990) et par un questionnaire sur les habitudes alimentaires de ces populations.

Deux populations peuvent être différenciées dans les valeurs inférieures à $0,4 \mu \mathrm{g} \mathrm{l}^{-1}$ en fonction de la technique de mesure adoptée (Tab. 4). 


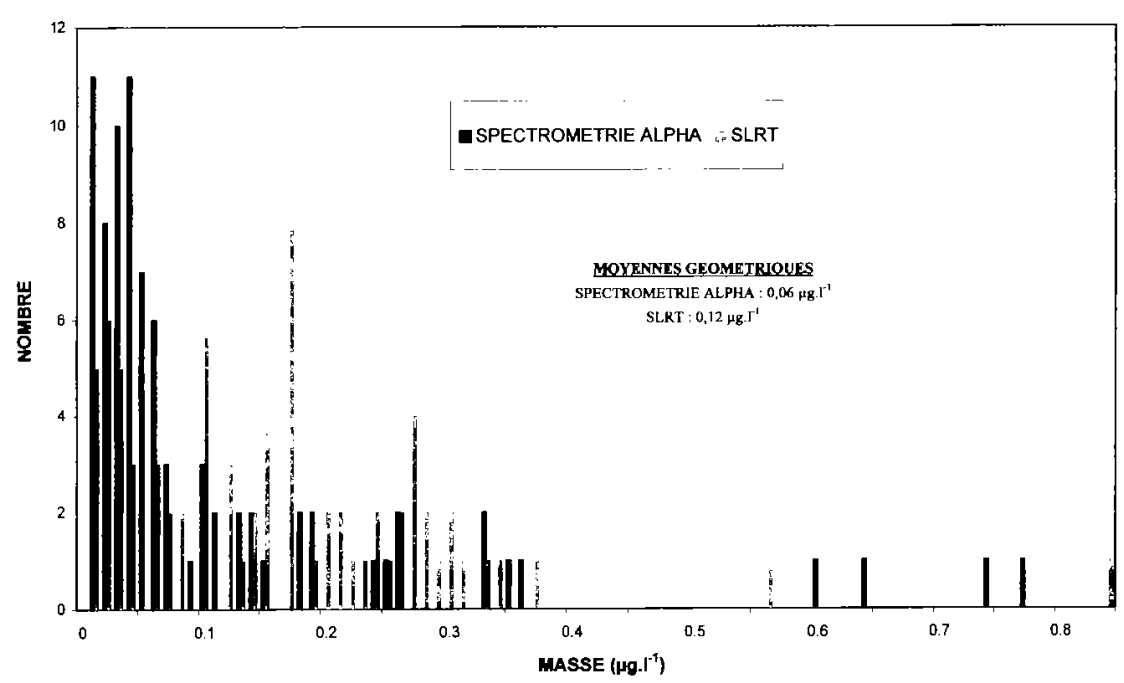

Fig. 7 - Valeur de l'excrétion urinaire de l'uranium chez une population de travailleurs non-exposés. Urinary uranium excretion data (non-exposed workers).

\section{TABLEAU IV}

Excrétion urinaire de l'uranium en fonction de la technique de mesure (travailleurs non-exposés).

Urinary excretion of uranium as a function of the measurement technique (non-exposed workers).

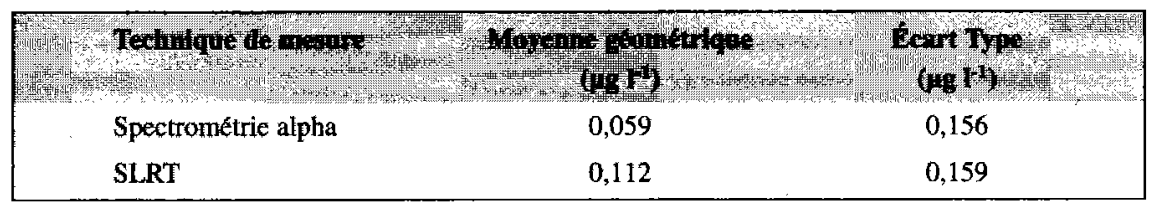

Les valeurs obtenues sont en bon accord avec les données bibliographiques, bien que des variations significatives puissent être mises en évidence selon le pays, la région concernée et selon les méthodes de dosage mises en œuvre (Dang, 1989; Dang, 1992; Harduin, 1994; Michaud, 1985; Shiraishi, 1992; Singh, 1990; Wrenn, 1985; Wrenn, 1992). 


\section{Conclusions}

\subsection{Inconvénients}

La SLRT ne fournissant aucune information sur la composition isotopique du contaminant, celle-ci doit évidemment être complétée par les mesures radiochimiques avec spectrométrie alpha, quand celles-ci s'avèrent nécessaires pour l'interprétation radiotoxicologique des résultats (principalement lorsque le risque radiologique prime sur le risque chimiotoxique).

\subsection{Avantages}

La SLRT présente l'avantage fondamental, par rapport aux autres techniques de dosage rapide de l'uranium, de pouvoir visualiser et enregistrer un spectre caractéristique de fluorescence sans interférence des autres actinides, dans les conditions opératoires de dosage pratiquées.

Bien que les limites de détection obtenues soient plus élevées que celles obtenues par activation neutronique, la SLRT présente l'avantage d'une portabilité plus importante et prend en compte tous les isotopes de l'uranium (isotopes fissiles et fertiles).

La SLRT permet de fournir un résultat rapide (premier résultat en 45 minutes, puis un résultat toutes les 5 minutes en cas d'échantillons multiples). Ce délai de réponse est à comparer à celui de la spectrométrie alpha et à celui de l'activation neutronique (plusieurs jours).

Les délais de réponse de la mesure par SLRT après minéralisation ( 2 jours) et ceux de la mesure rapide après traitement au charbon actif (45 minutes) doivent être pris en compte en fonction du degré d'urgence demandé pour la mesure de l'uranium. En fonction des deux critères, limite de détection et rapidité de réponse, le choix s'orientera préférentiellement vers l'une ou l'autre des stratégies, en ne perdant pas de vue que la mesure rapide peut être confirmée si nécessaire par une mesure différée à la fois par SLRT et par spectrométrie alpha sur des aliquotes du même prélèvement minéralisé.

\subsection{Performances}

Le dosage en urgence de l'uranium urinaire est possible avec une incertitude et une précision de l'ordre de $10 \%$. Ce résultat convient parfaitement pour l'interprétation du risque chimiotoxique de l'uranium en cas de contamination aiguë par 
inhalation. La limite de détection de $2 \mu \mathrm{g} \mathrm{l}^{-1}$ obtenue pour les dosages en urgence avec des temps de mesure brefs ( 90 secondes) est bien adaptée aux objectifs de la surveillance des travailleurs exposés quelle que soit la classe radiotoxicologique des composés de l'uranium.

Le dosage différé sur des minéralisats d'urine permet d'atteindre une limite de détection de $0,1 \mu \mathrm{g} \mathrm{l}^{-1}$ pour des temps de mesure de 30 secondes. Ces performances permettent de mesurer les limites dérivées d'excrétion de l'uranium telles qu'elles ont été définies par la Commission Internationale de Protection Radiologique (ICRP, 1979; ICRP, 1988).

Des temps d'intégration de la mesure beaucoup plus prolongés permettent d'atteindre des limites de détection beaucoup plus basses, qui ne s'imposent pas dans le cas précis de la surveillance de la contamination interne des travailleurs exposés.

\section{RÉFÉRENCES}

Berthoud T., Decambox P., Kirsch B., Mauchien P., Moulin C. (1988) Direct uranium trace analysis in plutonium by time-resolved laser-induced spectrofluorimetry. Anal. Chem., 60, 1296-1299.

Dang H.S., Pullat V.R., Jaiswall D.D., Parameswaran M., Sunta C.M. (1989) Daily intake of uranium by urban indian population. I. Radioanal. Nucl. Chemistry art, 26, 119-122.

Dang H.S., Pullat V.R., Pillai K.C. (1992) Determining the normal concentration of uranium in urine and application of the data to its biokinetics. Health Phys., 62, 562-566.

Decambox P., Mauchien P., Moulin C. (1991) Direct and fast determination of uranium in human urine samples by laser-induced time-resolved spectrofluorimetry. Appl. Spectrosc., 45, 116-118.

Deniau H., Decambox P., Mauchien P., Moulin C. (1993) Time-resolved laser-induced spectrofluorimetry of $\mathrm{UO}_{2}^{2+}$ in nitric acid solutions. Preliminary results for on-line monitoring applications. Radiochim. Acta, 61, 23-28.

Fuger J., Khodakovsky I.L., Sergeyeva E.I., Medvediev V.A., Navratil J.D. (1992) in: The Chemical Thermodynamics of Actinide Elements and Compounds, The Actinide Aqueous Inorganic Complexes. AlEA Publications, 12, 163.

Grenthe. L., Fuger J., Lemire R.J., Muller A.B., Nguyen Trung C., Wanner H. (1990) Chemical Thermodynamics of uranium, NEA-TDB, OCDE Nuclear Energy Agency Data Bank, Final draft.

Harduin J.C., Royer P. Piechowsky J. (1994) Uptake and urinary excretion of uranium after oral administration in man. Radiat. Prot. Dosimetry, 53, 245-248.

Horwitz E.P. et al. (1990) Concentration and separation of actinides from urine using a supported bifunctional organophosphorus extractant. Analyt. Chim. Acta, 28, 291-304.

Horwitz E.P. et al. (1992) Separation and preconcentration of uranium from acidic media by extraction chromatography. Analyt. Chim. Acta, 266, 25-27.

CIPR (1979) Limits for intakes of radionuclides by workers, ICRP Publication 30., Pergamon Press: Oxford, 3, $\mathrm{N}^{\circ} 1-4$.

CIPR (1988) Individual monitoring for intakes of radionuclides by workers: Design and interpretation, ICRP publication 54, Pergamon press: Oxford, 19, $\mathrm{N}^{\circ} 1-3$.

Michaud F., Archimbaud M., Chalabreysse J. (1985) Evvaluation de la teneur en uranium naturel des urines et des selles chez des sujets non-exposés professionnellement. Radioprotection, 20, 345346. 
Moulin C., Beaucaire C., Decambox P., Mauchien P. (1990) Determination of uranium in solution at the $\mathrm{ng} \mathrm{L}^{-1}$ level by time-resolved laser-induced spectrofluorimetry: application to geological samples. Anal. Chim. Acta, 238, 291-296.

Moulin C., Decambox P., Couston L., Pouyat D. (1994) Time-resolved laser-induced fluorescence of - $\mathrm{UO}_{2}^{2+}$ in nitric solutions. Comparison between nitrogen and tripled Nd-YAG laser. J. Nuc. Science Techn., 31, 691-699.

Moulin C., Decambox P., Moulin V., Decaillon J.G. (1995) Uranium speciation in solution by timeresolved laser-induced fluorescence. Anal. Chem., 34, 348-353.

Nelson D.M., Larosa J.J., Fairman W.D., Horwitz E.P., Dietz M.L. (1990) Concentration and separation of actinides from urine using a supported bifunctional organophosphorus extractant. Analyt. Chim Acta, 238, 263-271.

Remy M.L., Lemaitre N. (1990) Eaux minérales et radioactivité. Hydrogéologie, 4, 267-278.

Shiraishi K., Igarashi Y., Takaku Y. (1992) Daily intakes of ${ }^{232} \mathrm{Th}$ and ${ }^{238} \mathrm{U}$ in Japanese males. Health Physics, 63, 187-191.

Still C. W. (1974) Purification of radioactive tracers for use in high sensitivity alpha spectrometry. Anal. Chem., 46, 1426-1431.

Singh P., Ruth P., Burleigh P., Wrenn M.E. (1990) Daily uranium in Utah residents from food and drinking water. Health Physics, 59, 333-337.

Wrenn M.E., Durbin P., Howard B., Lipszein J., Rundo J., Still E., Willis D. (1985) Metabolism of ingested uranium and radium. Health physics, 48, 601-633.

Wrenn, M.E., Ruth H., Burleigh P., Singh P. (1992) Background levels of uranium in human urine. J. Radioanal. Nucl. Chemistry Art, 156, 407-412. 